\title{
Infectious Diseases Threat and Africa's Health Security
}

\author{
John Nkengasong, ${ }^{1 *}$ Richard Kamwi ${ }^{2}$ \\ ${ }^{1}$ Africa Centres for Disease Control and Prevention, Addis Ababa, Ethiopia \\ ${ }^{2}$ Ambassador, Southern Africa Malaria Elimination and Initiative, Windhoek, Namibia and African Union Champion of the Africa Centres for \\ Disease Control and Prevention \\ *Corresponding author, email: NkengasongJ@africa-union.org
}

In the 1970s, because of the remarkable progress made in the areas of vaccines and antibiotics, public health officials felt confident that the threats posed by infectious diseases could be eliminated. However, the emergence of infectious diseases, such as the human immunodeficiency virus, in the 1980s challenged this confidence. More recently, the world has observed the emergence and re-emergence of infectious diseases with pandemic potentials. Africa bears the highest burden of infectious disease pathogens in the world but has the weakest public health systems and infrastructure. This situation poses a severe security and economic threat to the continent. For instance, the Ebola virus disease (EVD) outbreak in West Africa killed an estimated 11000 people and reversed gains made in economic growth in the affected countries.

Social and environmental determinants related to urbanisation, increases in population and mobility have created conducive conditions for the spread of infectious diseases. In response, for example, a grouping of eight countries within the Southern Africa Development Community have set aggressive targets to eliminate the local transmission of malaria. However, Zimbabwe, South Africa, and Swaziland recorded increases of $70 \%, 160 \%$, and $48 \%$ respectively during the first half of 2017 , as compared to the first half of 2016. A concerning trend in this region is the re-emergence of malaria in districts that had for long been considered malaria free - the result of population displacement, as well as changing ecological and climatic factors.

The population of Africa is expected to increase from 1.2 billion to 2.5 billion people by the year 2050 . Additionally, the widespread use of medications has created an enormous threat of the emergence of antimicrobial resistance. To address these challenges, the leadership of Africa has taken two significant actions: Firstly, in January 2017, the heads of states and governments of Africa launched the Africa Centres for Disease Control and Prevention (Africa CDC), in recognition of the social, economic, and security dimensions of infectious disease threats. The Africa CDC's mission is to support Member States to strengthen their public health capacity. The work of Africa CDC will initially focus on five pillars: 1) disease surveillance and intelligence, 2) pandemic preparedness and response, 3) laboratory networks, 4) innovative information systems, and 5) public health institutes and research. Africa CDC will support Member States to establish or strengthen National Public Health Institutes (NPHIs) and link them to the five regional collaborating centres in Egypt, Nigeria, Gabon, Zambia, and Kenya. Africa CDC has also established the Regional Integrated Surveillance and Laboratory Networks (RISLNET) in order to ensure that all existing public health assets are efficiently used in the region to respond to disease needs. The five regional centres will be linked to the headquarters in Addis Ababa. Secondly, in July 2017, African heads of states and governments issued a declaration to accelerate the implementation of international health regulations (IHR) on the continent. This declaration offers a fresh impetus and opportunity for strengthening public health systems on the continent, required to ensure the health security of Africa. However, in order to successfully accelerate the implementation of IHR, a multi-sectorial approach will be required: 1) Member States will need to commit financial resources to improve the public health systems and infrastructure including strengthening public health surveillance and laboratory systems and networks, the use of standard case definitions, improvement of data sharing across the continent, and strengthening of public health research and human resources for health; 2) an assessment system, such as the joint external evaluation, must be implemented in a systematic manner to allow for an objective and accountable methodology to measure progress made by Member States in strengthening gaps in their national health systems for IHR; 3) the private sector will need to be fully engaged, given the economic threat that diseases pose to businesses; and 4) donors and technical partners will need to coordinate their efforts closely with those of the Member States, Africa CDC, and the World Health Organization to ensure a unified effort.

In closing, because of globalisation, a disease outbreak or threat anywhere in Africa is clearly a security and economic menace everywhere in the world. As such, the prevention and control of outbreaks require public health institutions in Africa to work in a network, such as the RISLNET, in order to harness the assets that exist across the continent to better respond to disease threats. 